\title{
Chronic Hyponatremia Reduces Survival of Magnocellular Vasopressin and Oxytocin Neurons after Axonal Injury
}

\author{
Janos Dohanics, ${ }^{1}$ Gloria E. Hoffman, ${ }^{2}$ and Joseph G. Verbalis ${ }^{3}$ \\ Departments of ${ }^{1}$ Medicine and ${ }^{2}$ Neurobiology, University of Pittsburgh, Pittsburgh, Pennsylvania 15261, and \\ 3Department of Medicine, Georgetown University, Washington, DC 20007
}

\begin{abstract}
Axonal injury to hypothalamic magnocellular vasopressin (AVP) and oxytocin (OT) neurons causes degeneration of a substantial subpopulation of these neurons. In this study, we investigated the influence of osmolality on this injury-induced cell death. Normonatremic, chronically hypernatremic, and chronically hyponatremic rats received pituitary stalk compression (SC), which causes degeneration of AVP and OT terminals in the neurohypophysis. Twenty-one days after SC, rats were perfused and hypothalami were serially sectioned and alternately stained for AVPneurophysin and OT-neurophysin immunoreactivities. Normonatremic and hypernatremic rats exhibited a triphasic pattern of water intake after SC, with peak intakes 3 times higher than those exhibited by sham-operated normonatremic rats. In contrast, hyponatremic SC rats exhibited peak water intakes of $600 \mathrm{ml} / 24 \mathrm{hr}$, $\sim 9-10$ times the water intakes of sham-operated normonatremic
\end{abstract}

rats. In normonatremic rats, SC caused degeneration of $65 \%$ of the AVP neuron population in the SON and $73 \%$ in the PVN, but only $31 \%$ of the OT neuron population in the SON and $35 \%$ in the PVN. Similar results were found in hypernatremic rats after SC. However, in hyponatremic rats SC caused degeneration of $97 \%$ of the AVP neuron population in the SON and 93\% in the PVN, and $90 \%$ of the OT neuron population in the SON and $84 \%$ in the PVN. Our results, therefore, demonstrate that injury-induced degeneration of magnocellular AVP and OT neurons is markedly exacerbated by chronic hypo-osmolar conditions, but neuronal survival is not enhanced by chronic hyperosmolar conditions.

Key words: axonal injury; vasopressin neurons; oxytocin neurons; osmolality; neuronal death; hyponatremia; diabetes insipidus
Injury to magnocellular vasopressin (AVP) and oxytocin (OT) neurons induces marked changes in the morphology and function of the neurohypophysis. Neurolobectomy and transection of the pituitary stalk result in degeneration of the neural lobe of the pituitary gland and the development of diabetes insipidus (O'Connor, 1952; Moll and De Wied, 1962). In addition, disruption of the axons of the hypothalamo-neurohypophyseal tract also leads to retrograde degeneration of substantial numbers of magnocellular neurons in the supraoptic (SON) and paraventricular (PVN) nuclei of the hypothalamus (Hare, 1937; Raisman, 1973).

Degeneration of the perikaryon after axonal damage is not unique to magnocellular neurons, because this phenomenon has been observed throughout the CNS (Fry and Cowan, 1972; Brand and Mugnaini, 1976; Kawaguchi et al., 1981; Sofroniew and Isacson, 1988). However, only a few studies to date have ascertained possible factors influencing the survival of axonally damaged CNS neurons. Changes in hormonal milieu (Yu, 1989) and the volume of the axoplasma separated from the perikaryon (Fry and Cowan, 1972; Sofroniew and Isacson, 1988) have been suggested as factors influencing survival after axonal injury. Neurotrophic factors such as fibroblast growth factor (Grothe et al., 1989) and nerve growth factor (Hefti, 1986; Tuszynski et al., 1990), as well as fetal brain grafts (Sievers et al., 1989), have been reported to rescue CNS neurons from axotomy-induced degeneration. Fetal hypothalamic grafts have also been reported to rescue hypothalamic magnocel-

Received Jan. 2, 1996; revised Jan. 10, 1996; accepted Jan. 11, 1996.

These studies were supported in part by National Institutes of Health Grants DK02014 (J.D.) and DK38094 (J.G.V.).

Correspondence should be addressed to Dr. Joseph G. Verbalis, 232 Building D, Georgetown University, 4000 Reservoir Road NW, Washington, DC 20007.

Copyright 1996 Socicty for Neuroscience $0270-6474 / 96 / 162373-08 \$ 05.00 / 0$ lular neurons after neurohypophysectomy (Marciano et al., 1989). In contrast, Herman et al. (1986) reported that AVP administration to neurolobectomized rats was associated with an increased rate of degeneration of magnocellular AVP neurons and prevented functional recovery of the vasopressinergic system (Herman et al., 1987). Interestingly, however, this treatment appeared to have little effect on the survival of magnocellular oxytocinergic neurons, which were not affected after neurolobectomy, as assessed by visual analysis.

Recently, we have characterized the effects of a brief $(30 \mathrm{sec})$ compression of the pituitary stalk in rats, which include degeneration of the posterior pituitary and development of diabetes insipidus, but not damage to the anterior pituitary (Dohanics et al., 1992). Similar to neurolobectomy and transection of the pituitary stalk, stalk compression (SC) also caused degeneration of magnocellular neurons, which was apparent by a visible decrease in the number of AVP, but not OT, neurons by immunocytochemical staining of hypothalamic sections. In this study, hypothalamic AVP and OT neurons were counted after SC to provide a more quantitative assessment of the effects of axonal injury on survival of magnocellular neurons. In addition, we performed a similar analysis on rats that were made chronically hypo- or hypernatremic before SC, treatments known to downregulate and upregulate neurohypophyseal secretion (Claybauch, 1976; Verbalis et al., 1986; Verbalis and Dohanics, 1991) and synthesis (Sherman et al., 1986; Robinson et al., 1990), respectively, to evaluate whether the functional activity of magnocellular neurons before $\mathrm{SC}$ affected their subsequent survival.

Parts of this work were presented in abstract form at the 20th Annual Meeting of the Society for Neuroscience (St. Louis, MO, 1990). 


\section{MATERIALS AND METHODS}

\section{Animals}

Adult male Spraguc-Dawley rats weighing 250-275 gm (Zivic-Miller, Allison Park, PA) were housed individually in wire-mesh cages in a temperature-controlled room $\left(21-23^{\circ} \mathrm{C}\right)$ with lights on from 7:00 A.M. to 7:00 P.M. Animals were fed solid food or liquid diet as described below. Daily fluid consumption was recorded throughout all studies.

\section{Pituitary stalk compression}

Stalk compression was performed as described previously (Dohanics et al., 1992). Briefly, rats were anesthetized with methoxyflurane (Metofane, Pitman-Moore, Washington Crossing, NJ) and mounted on a stereotaxic frame, then the skull was opened through an $\sim 3 \mathrm{~mm}$ by $2 \mathrm{~mm}$ window. A triangle-shaped wire $(\sim 1.5 \mathrm{~mm}$ wide at the base $)$ was lowered in the coronal plane $4.0 \mathrm{~mm}$ caudal to bregma in the midline until it touched the floor of the skull. The wire was held against the skull for $30 \mathrm{sec}$ and then removed. For sham surgeries, the wire was lowered $9 \mathrm{~mm}$ beneath the surface of the brain. The wound was closed, and rats received $60,000 \mathrm{U}$ of penicillin $G$ (i.m.).

\section{Induction of hypernatremia and hyponatremia}

To induce hypernatremia, rats were given $2 \% \mathrm{NaCl}$ solution ad libitum as their only drinking fluid for $7 \mathrm{~d}$. Daily saline intake was monitored. Hyponatremia was induced as described previously (Verbalis and Drutarosky, 1988). Briefly, rats were fed with $40 \mathrm{ml} / \mathrm{d}(70 \mathrm{kcal} / \mathrm{d})$ of a nutritionally balanced liquid diet (AIN-76, Bioserv, Frenchtown, NJ), which was presented daily each morning. After $2 \mathrm{~d}$ on liquid diet, osmotic minipumps (Alzet model 2002, Alza, Palo Alto, CA) containing 1-desamino-[8-D-arginine]-vasopressin (DDAVP; Rorer Pharmaceuticals, Fort Washington, PA) were implanted subcutancously using methoxyfluranc ancsthesia to deliver DDAVP at a rate of $5 \mathrm{ng} / \mathrm{hr}$. On the day of osmotic minipump implantation, the rats were given a more diluted preparation of the liquid diet $(70 \mathrm{kcal}$ in $60 \mathrm{ml})$, but thereafter resumed the more concentrated formula $(70 \mathrm{kcal}$ in $40 \mathrm{ml})$.

\section{Treatment groups}

The following four groups of rats were studied.

Sham-operated normonatremic rats. These rats received sham stalk compression and had access to either solid chow ad libitum or $40 \mathrm{ml}$ of liquid diet daily. They also had unrestricted access to tap water, the consumption of which was monitored daily.

Stalk-compressed nomonatremic rats. These rats received SC and had access to either solid chow ad libitum or $40 \mathrm{ml}$ of liquid diet daily. They also had unrestricted access to tap water, the consumption of which was monitored daily.

Stalk-compressed hypernatremic rats. These rats were given $2 \% \mathrm{NaCl}$ solution ad libitum as their only drinking fluid for $7 \mathrm{~d}$. On the seventh day, these rats received SC. After the surgery, they were given tap water instead of $2 \% \mathrm{NaCl}$. Water and $2 \% \mathrm{NaCl}$ intakes were monitored daily. These rats had unrestricted access to solid food.

Stalk-compressed hyponatremic rats. Seven days after the induction of hyponatremia, these rats received SC. Hyponatremia was then maintained for the next $7 \mathrm{~d}$, after which the rats were allowed to return to normonatremia. Because rapid correction of chronic severe hyponatremia is known to cause severe neurological effects (Verbalis et al., 1991a), rats were slowly made normonatremic over several days to avoid such side effects. Seven days after surgery ( $14 \mathrm{~d}$ after the induction of hyponatremia), the DDAVP infusion was terminated by removing the osmotic minipumps, but the rats remained on the liquid diet for an additional $2 \mathrm{~d}$ before they were given solid food ad libitum.

On the day of the SC surgery, a blood sample was drawn from all rats via jugular puncture for measurement of plasma sodium concentrations $\left(\left[\mathrm{Na}^{+}\right]\right)$using an ion-specific electrode (Beckman Electrolyte 2 Analyzer, Brea, CA).

\section{Histology}

Twenty-one days after SC surgery, rats received a lethal dose of pentobarbital $(100 \mathrm{mg} / \mathrm{kg})$. After an intracardiac injection of heparin $(300 \mathrm{U})$, rats were perfused through the ascending aorta with $0.9 \% \mathrm{NaCl}$ containing $2 \%$ sodium nitrite (total volume, $200 \mathrm{ml}$ ). The perfusion solution was then switched to a fixative consisting of $4 \%$ paraformaldehyde and $1.4 \%$ picric acid in $0.1 \mathrm{M}$ phosphate buffer (total volume, $100 \mathrm{ml}$ ). Perfused rats were decapitated, and the brains and pituitaries were stored in $25 \%$ sucrose at $4^{\circ} \mathrm{C}$ until sectioning. Hypothalami were cut with a freezing microtome into $25 \mu \mathrm{m}$ sections in the coronal plane. Alternate sections were processed for AVP-neurophysin (AVP-NP) and OT-neurophysin (OT-NP) immunohistochemical stainings using antisera produced by immunizations with $\mathrm{N}$-terminal fragments of these peptides (Verbalis et al., 1991b). Stained sections were mounted on glass slides and coverslipped. Pituitaries were stored in $25 \%$ sucrose for $24 \mathrm{hr}$ at $4^{\circ} \mathrm{C}$ and then immersed in $15 \%$ gelatin (J. T. Baker Chemical Company, Phillipsburg, $\mathrm{NJ}$ ) for $15 \mathrm{~min}$ at $37^{\circ} \mathrm{C}$. Gelatin-embedded pituitaries were fixed in the perfusion solution for $24 \mathrm{hr}$ at $4^{\circ} \mathrm{C}$ and then stored in $25 \%$ sucrose for 24 $\mathrm{hr}$ at $4^{\circ} \mathrm{C}$. Pituitaries were cut with a freezing microtome into $40 \mu \mathrm{m}$ sections. Sections were stained for AVP-NP and OT-NP immunoreactivities. Some hypothalamus and pituitary sections were stained with cresyl violet. Rats with incomplete SC (as determined by pituitary histology) were excluded from further evaluation.

\section{Cell counting}

AVP-NP- and OT-NP-immunoreactive cells in the PVN and SON were counted on all scctions. Neurons werc counted at $40 \times$ magnification by identifying them visually or by visual identification combined with mapping of the position of identified neurons. The position of individual neurons was recorded using a microscope stage connected to a Macintosh computer that recorded the $x-y$ coordinates of the stage position. In each group, neurons in sections with cresyl violet staining only were also counted over an $80 \times 80 \mu \mathrm{m}^{2}$ area of the SON $(n=4)$. Raw counts were corrected for double-counting errors using Abercrombie's method (Abercrombie, 1946)

\section{Data analysis}

All results are exprcssed as mean \pm SE. Statistical analysis was performed on logarithmic transforms of the data using ANOVA followed by Tukey's protected $t$ tests for multiple comparisons where appropriate.

\section{RESULTS}

Plasma $\left[\mathrm{Na}^{+}\right]$of the hypernatremic and hyponatremic rats at the time of SC was significantly higher and significantly lower, respectively, than in normonatremic rats (Table 1).

Water intake of the sham-operated normonatremic rats remained relatively constant over the entire period of observation. As reported previously, stalk-compressed normonatremic rats exhibited a triphasic pattern of water intake: a sharply increased intake during the first $24 \mathrm{hr}$ after surgery (phase 1), followed by 2-3 d of water intake levels comparable with those observed in sham-operated rats (phase 2), after which water intake increased again and remained elevated throughout the remainder of the observation period (phase 3) (Fig. 1).

Stalk-compressed hypernatremic rats that drank $2 \% \mathrm{NaCl}$ only during the $7 \mathrm{~d}$ immediately preceding SC consumed increasingly larger amounts of the saline solution during that period. The water intake of the hypernatremic rats during the first $24 \mathrm{hr}$ after SC was significantly higher $(p<0.01)$ than that of sham-operated rats during the same period and, thereafter, the stalk-compressed hypernatremic rats exhibited a pattern of water intake similar to that of SC normonatremic rats (Fig. 2).

As expected, the stalk-compressed hyponatremic rats consumed very small amounts of water as long as hyponatremia was maintained, and stalk compression did not have any immediate effect on the water intake of these rats during this period. However, water intake began to increase from day 8 after SC, the first day

Table 1. Plasma $\left[\mathrm{Na}^{+}\right](\mathrm{mmol} / \mathrm{l}$; mean $\pm \mathrm{SE})$ at the time of surgery

Sham-operated SC/normonatremic SC/hypernatremic SC/hyponatremic

$\begin{array}{cccc}144.1 \pm 0.8 & 142.4 \pm 1.0 & 179.7 \pm 4.5 & 103.9 \pm 0.6 \\ (6) & (7) & (7) & (6) \\ & \text { NS } & \dagger \dagger & \text { 十† }\end{array}$

NS, $p>0.05$ compared with sham-operated; $+\dagger, p<0.001$ compared with $\mathrm{SC} /$ normonatremic by one-way ANOVA. 


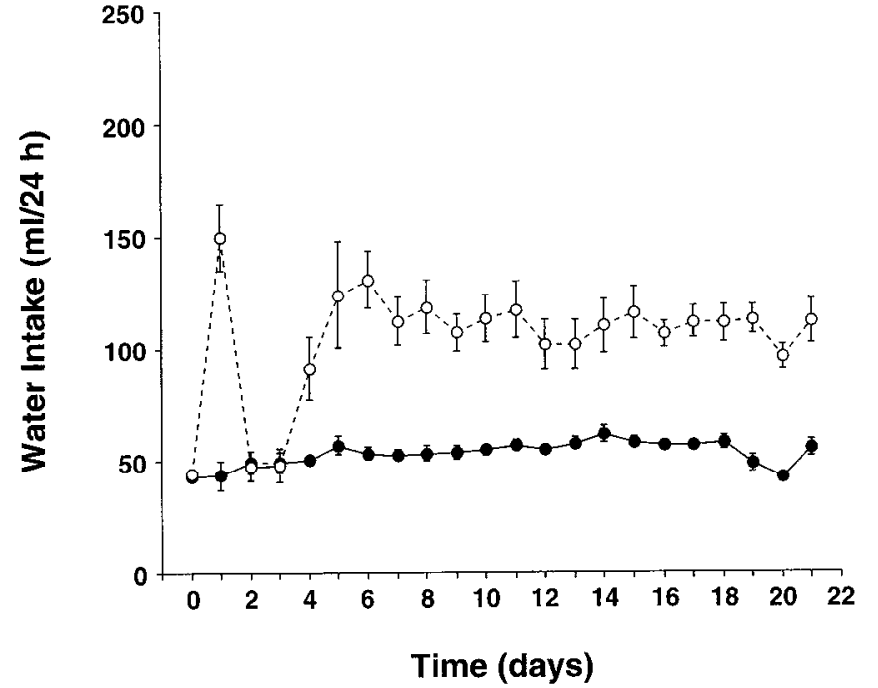

Figure 1. Water intake of sham-operated (closed circles; $n=6$ ) and SC rats (open circles; $n=7$ ). Mean $\pm \mathrm{SE}$ are shown.

after the termination of DDAVP infusion. During the subsequent days, water intake then increased further to extremely high levels, with peak intakes often exceeding $600 \mathrm{ml} / 24 \mathrm{hr}$. The rate of increase in water intake varied considerably in individual rats, peaking as early as $12 \mathrm{~d}$ after SC $(5 \mathrm{~d}$ after the cessation of DDAVP infusion) or as late as $15 \mathrm{~d}$ after SC $(8 \mathrm{~d}$ after the cessation of DDAVP infusion). Because the water intake curves of these rats were shifted by several days, individual examples are shown in Figure 3 instead of the group means. However, the mean peak water intake of the stalk-compressed hyponatremic rats was markedly higher than that of the stalk-compressed normonatremic $(p<0.001)$ and hypernatremic rats (Fig. 4).

In normonatremic rats, SC caused degeneration of a significant portion of the AVP neuron population in both the SON and the PVN, resulting in the survival of only $\sim 35$ and $\sim 27 \%$ of AVP neurons in these nuclei, respectively (Figs. 5, 6; Table 2). In contrast, a much greater portion of the OT neuron population in the magnocellular nuclei survived $\mathrm{SC}, \sim 69 \%$ in the $\mathrm{SON}$ and $\sim 65 \%$ in the PVN (Figs. 7, 8; Table 2).

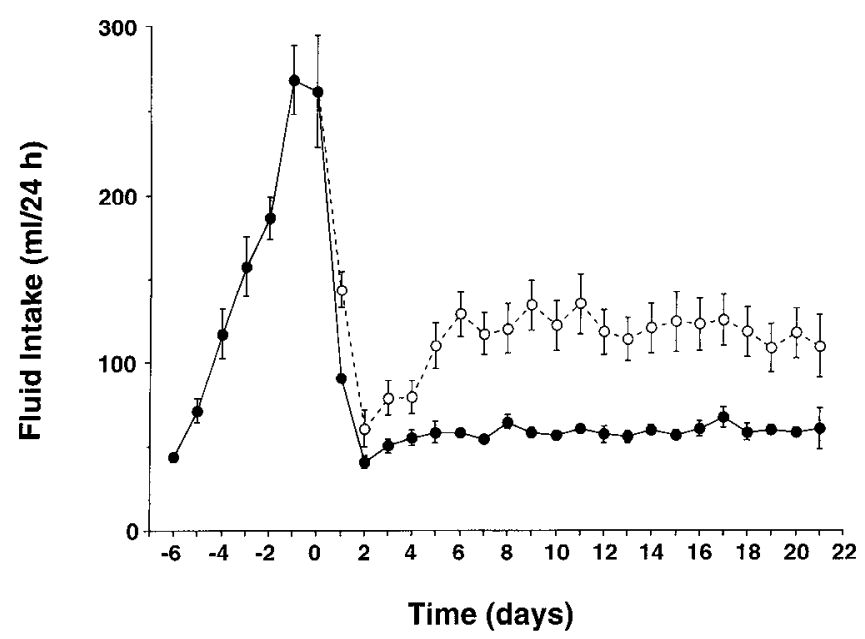

Figure 2. Fluid intake of sham-operated/hypernatremic (closed circles; $n=6$ ) and $\mathrm{SC} /$ hypernatremic rats (open circles; $n=7$ ). Before surgery on day 0 , all rats had access to $2 \% \mathrm{NaCl}$ only. After surgery, all rats had unrestricted access to tap water. Mean $\pm \mathrm{SE}$ are shown.

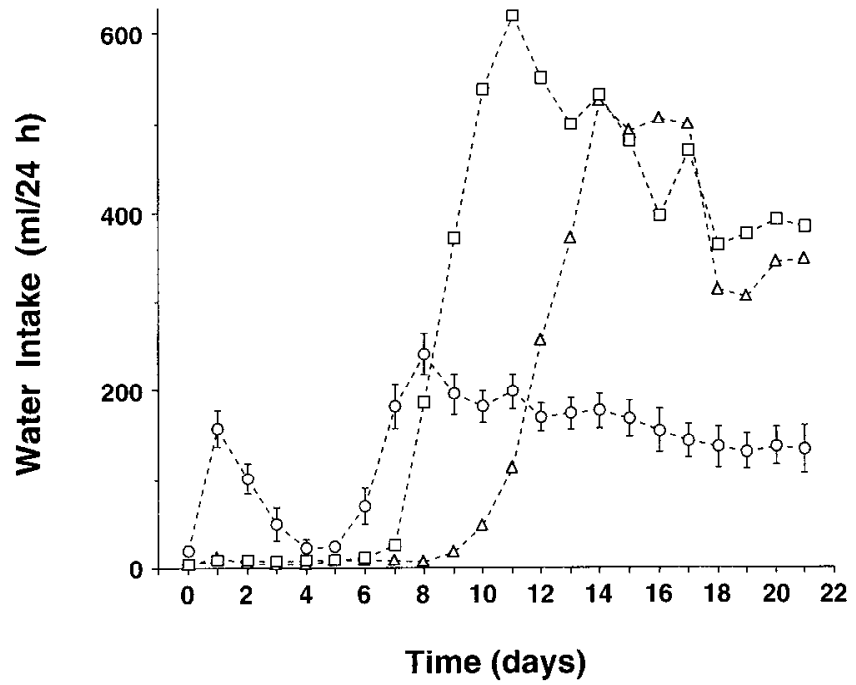

Figure 3. Water intake of $\mathrm{SC} /$ normonatremic rats (open circles; $n=6$; mean $\pm \mathrm{SE}$ ) and individual SC/hyponatremic rats (\#31: open triangles; \#38: open squares). The SC/normonatremic rats were fed the same liquid diet as the $\mathrm{SC} /$ hyponatremic rats but did not receive DDAVP infusions (see Materials and Methods).

In hypernatremic rats, the extent of magnocellular neuronal degeneration after SC was similar to that observed in normonatremic rats. The portion of the surviving AVP neuron population was $\sim 35$ and $\sim 23 \%$ in the SON and PVN, respectively (Figs. 5 , 6; Table 2), and the portion of the surviving OT neuron population was $\sim 61$ and $\sim 60 \%$, respectively (Figs. 7, 8; Table 2).

Hyponatremia greatly exacerbated the extent of magnocellular neuronal degeneration caused by SC. In the hyponatremic rats, only $\sim 3$ and $\sim 7 \%$ of the AVP neurons in the SON and in the PVN survived, respectively (Figs. 5, 6; Table 2). The survival rates of OT neurons in the SON and PVN also decreased significantly to $\sim 10$ and $-16 \%$, respectively (Figs. 7, 8; Table 2).

Counting of magnocellular neurons in sections stained with

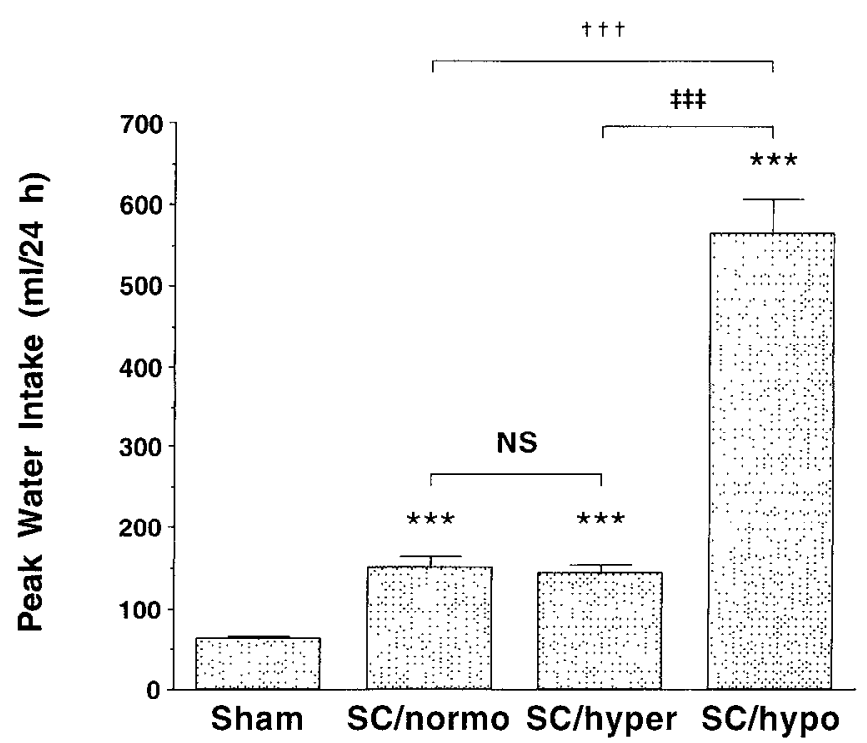

Figure 4. Maximum 24 hr water intake in sham-operated (Sham), stalkcompressed normonatremic (SC/nomo), stalk-compressed hypcrnatremic (SC/hyper), and stalk-compressed hyponatremic rats (SC/hypo). Mean \pm SE are shown. ${ }^{* * *}, p<0.001$ compared with sham; $N S, p>0.05 ;+\uparrow+, p<$ $0.001 ;$ 
A

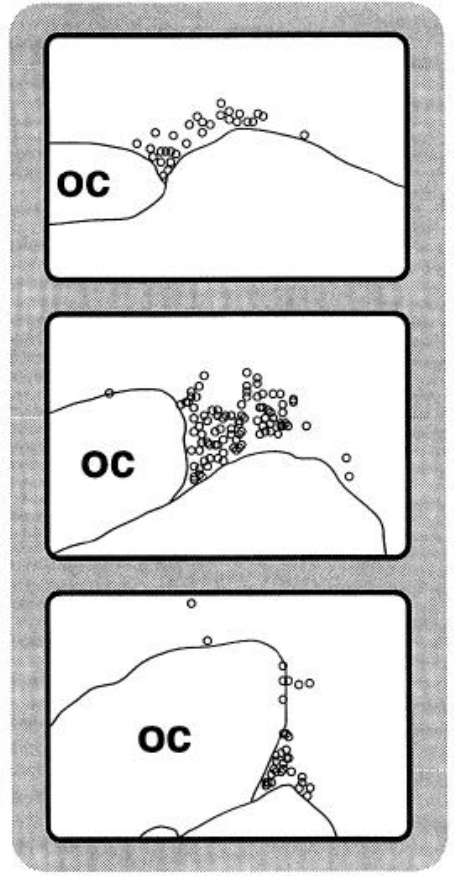

sham
B

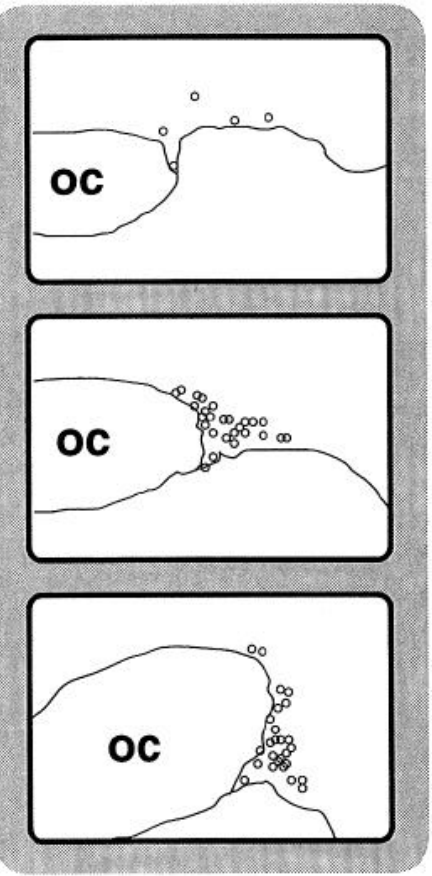

SC/normo
C
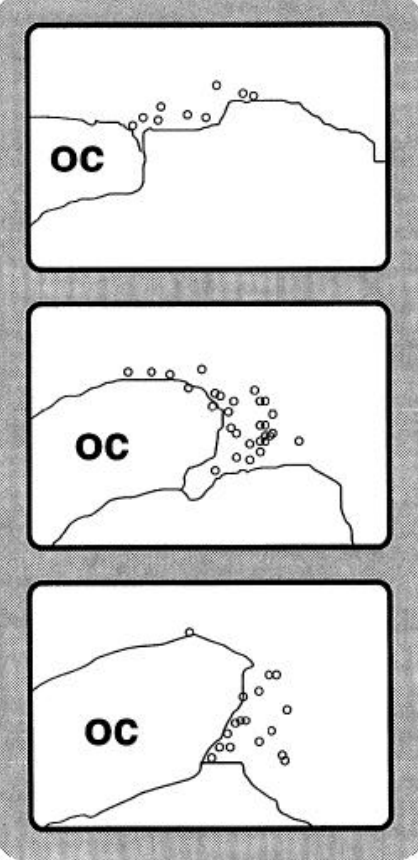

SC/hyper
D

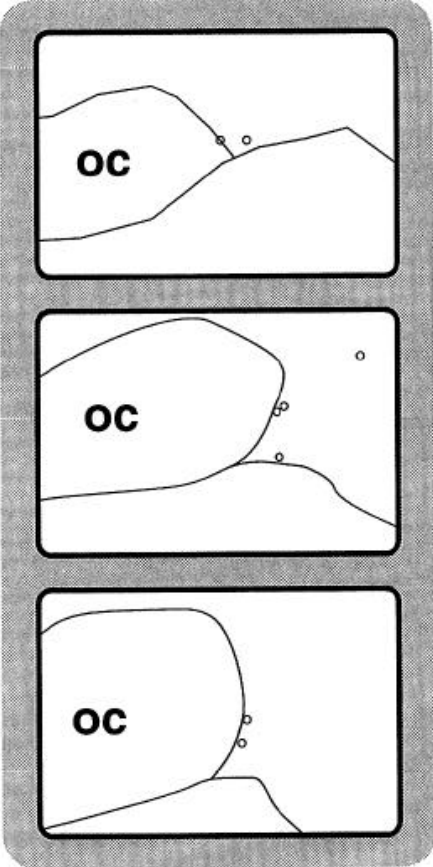

SC/hypo

Figure 5. Topographically correct representation of AVP-NP neurons at three different rostrocaudal levels in the SON from a normonatremic sham-operated rat $(A)$, a normonatremic SC rat $(B)$, a hypernatremic SC rat $(C)$, and a hyponatremic SC rat $(D)$. oc, Optic chiasm.

A

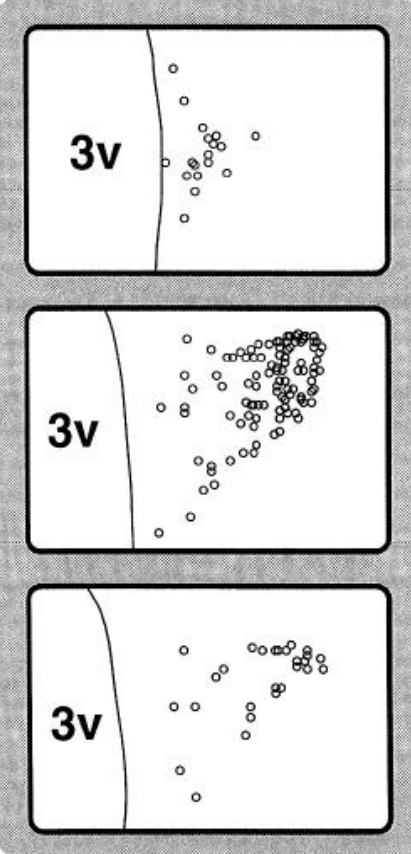

\section{sham}

B
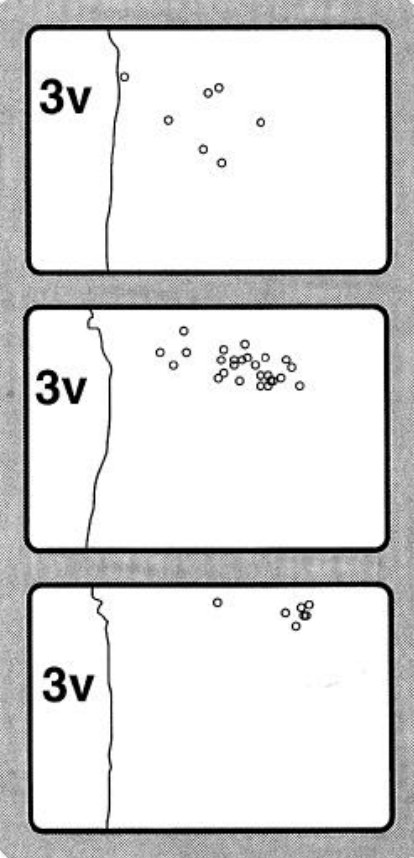

SC/normo
C
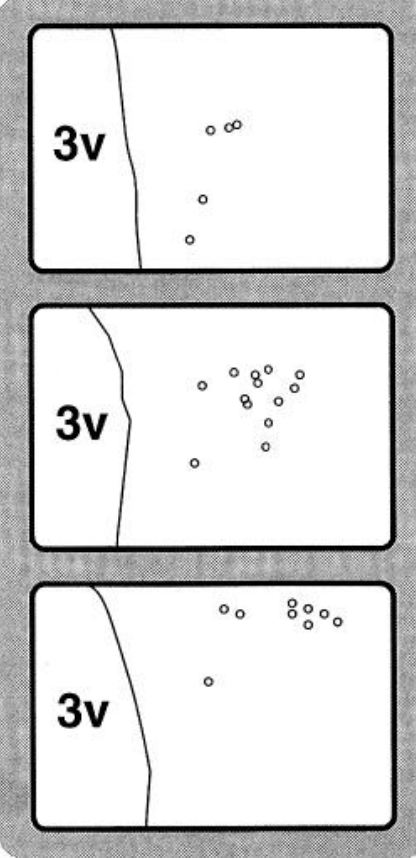

SC/hyper
D
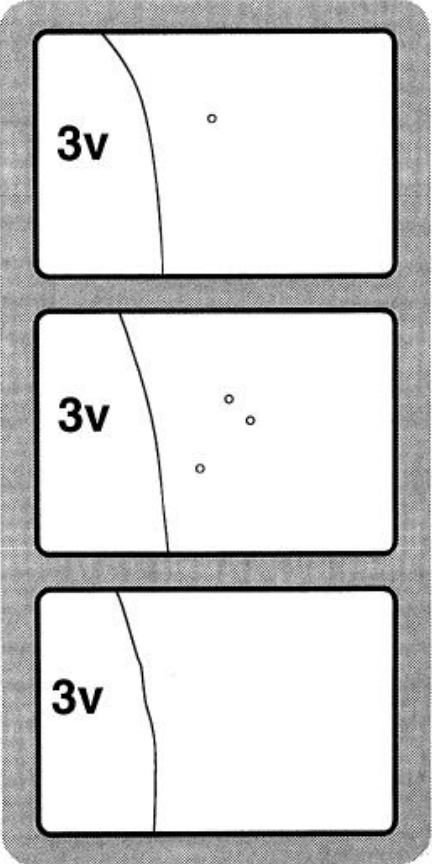

SC/hypo

Figure 6. Topographically correct representation of AVP-NP neurons at three different rostrocaudal levels in the PVN from a normonatremic sham-operated rat $(A)$, a normonatremic SC rat $(B)$, a hypernatremic SC rat $(C)$, and a hyponatremic SC rat $(D)$. $3 v$, Third ventricle. 
Table 2. Cell counts (mean \pm SE) of hypothalamic AVP and O'T neurons

\begin{tabular}{lccc}
\hline AVP neurons in the SON & & & \\
Sham-operated & $2672 \pm 268$ & $(6)$ & \\
SC/normonatremic & $936 \pm 102$ & $(7)$ & $* *$ \\
SC/hypernatremic & $948 \pm 279$ & $(7)$ & $* * *$ \\
SC/hyponatremic & $97 \pm 9$ & $(6)$ & $* * *+\dagger \dagger$ \\
AVP neurons in the PVN & & & \\
Sham-operated & $994 \pm 141$ & $(6)$ & \\
SC/normonatremic & $270 \pm 28$ & $(7)$ & $* * *$ \\
SC/hypernatremic & $229 \pm 41$ & $(7)$ & $* * *$ \\
SC/hyponatremic & $69 \pm 23$ & $(6)$ & $* * *+\dagger \dagger$ \\
OT neurons in the SON & & & \\
Sham-operated & $1958 \pm 212$ & $(6)$ & \\
SC/normonatremic & $1361 \pm 135$ & $(7)$ & $*$ \\
SC/hypernatremic & $1198 \pm 164$ & $(7)$ & $*$ \\
SC/hyponatremic & $205 \pm 9$ & $(6)$ & $* * *+\dagger \dagger$ \\
OT neurons in the PVN & & & \\
Sham-operated & $2417 \pm 161$ & $(6)$ & \\
SC/normonatremic & $1585 \pm 107$ & $(7)$ & $* * *$ \\
SC/hypcrnatrcmic & $1459 \pm 105$ & $(7)$ & $* * *$ \\
SC/hyponatremic & $392 \pm 36$ & $(6)$ & $* * *+\dagger \dagger$ \\
\hline
\end{tabular}

${ }^{*}, p<0.05 ;^{* *}, p<0.01{ }^{* * *}, p<0.001$ compared with sham-operated; $\dagger+, p<$ 0.001 compared with $\mathrm{SC} /$ normonatremic; the numbers in parentheses indicate the numbers of animals in each group.

cresyl violet only over limited areas of the SON revealed an $\sim 56 \%$ survival rate in normonatremic SC rats $(306 \pm 23$ vs $545 \pm 22$ in sham operated rats, $p<0.01)$. Corresponding magnocellular survival rates were $\sim 67 \%$ in hypernalremic SC rats $(366 \pm 29$, NS compared with normonatremic SC) and $\sim 17 \%$ in hyponatremic $\mathrm{SC}$ rats $(98 \pm 5, p<0.001$, compared with normonatremic $\mathrm{SC}$ ).

\section{DISCUSSION}

Retrograde degeneration of magnocellular neurons is well known to occur after hypophysectomy (Hare, 1937) or neurolobectomy (Bodian and Maren, 1951). Although magnocellular degeneration after axonal damage has also been demonstrated quantitatively (Bodian and Maren, 1951), these studies did not attempt to evaluate survival of AVP and OT neurons differentially. Only a recent report by Herman et al. (1987) using qualitative immunohistochemistry suggested that AVP rather than OT neurons are predominantly affected by axonal injury. Our quantitative results demonstrate that in normonatremic rats axonal injury affects the AVP neuron population more than twice as severely as it does the OT neuron population. Although this significant loss in the number of AVP neurons was also readily apparent by simple visual inspection, in our previous report in which magnocellular neurons were not counted (Dohanics et al., 1992) we were unable to detect any obvious effect of SC on the number of surviving OT neurons, similar to the results reported by Herman et al. (1987). The present study now shows that smaller but still significant degrees of degeneration also occur in hypothalamic OT neurons. It also shows that the apparent loss in the number of magnocellular neurons exhibiting AVP or OT staining reffects a decreased number of cells after surgery and likely is not the result of a temporary or permanent inability of magnocellular neurons to produce AVP- or OT-like immunoreactivity. This observation is in agreement with numerous earlier reports (O'Connor, 1947; Bodian and Maren, 1951; Raisman, 1973).

The factors that might account for the greater resilience of OT neurons are unknown, but this observation is consistent with several other studies that strongly suggest that OT neurons also regenerate more readily than AVP neurons. Oxytocin but not AVP content of the stalk-median eminence is significantly increased after SC (Dohanics et al., 1992), and sprouting of OT but not AVP fibers into the external zone of the median eminence in PVN-lesioned rats has been observed (Antoni et al., 1988). One possible factor conferring a greater survival advantage to OT neurons could be a higher basal metabolic activity. In support of this, synthesis and secretion of OT appears to be less inhibited than AVP under conditions of chronic hyponatremia (Robinson et al., 1990; Verbalis and Dohanics, 1991). Another potential factor could be a more robust reparative response. In this regard, we have observed recently that after SC, intense expression of the immediate carly gene product c-Jun occurred in magnocellular OT neurons, but staining intensity in the few c-Jun-expressing AVP neurons was much weaker (Dohanics et al., 1993b).

Although our experiments do not reveal the mechanism(s) responsible for the extent of neuronal degeneration caused by axonal injury, several previous studies have suggested various factors that influence the survival of injured neuronal populations. The first hypothesis put forward to explain retrograde degenerdtion after axonal injury of CNS neurons indicated the importance of surviving axon collaterals and the amount of axoplasma retained by the injured neuron. In an elegant experiment, Fry and Cowan (1972) demonstrated that the larger the axonal arborization separated from the perikaryon, the more likely that neurons of the lateral mamillary nucleus would dic. This likely accounts for the long-standing observation that transection of the hypothalamo-hypophyseal stalk at more rostral levels causes more extensive neuronal degeneration in magnocellular nuclei and greater degrees of diabetes insipidus (O'Connor, 1947). Although there may have been a small degree of variability in the placement of $\mathrm{SC}$ in these studies, this factor could not have caused the wide differences in the survival rates between AVP and OT neurons in the same animals, nor is it likely to account for the large differences in survival rates of all magnocellular neurons between nomonatremic and hyponatremic rats.

A previous study has also suggested that hormones such as anabolic steroids could also influence survival of neurons after injury (Yu, 1989), but because in our studies we used male rats exclusively, this factor also seems unlikely to account for the effects we observed. However, because the hyponatremic rats in our experiments were exposed to DDAVP, the possibility that this vasopressin analog itself influenced magnocellular neuronal survival cannot be excluded. Reports on the effects of AVP and DD AVP on magnocellular neuronal function have not been consistent. Although some investigators have reported decreased AVP secretory responses to physiological stimuli in rats treated with AVP or DDAVP (Cheng and North, 1989), others have found no such inhibitory effects (Lundin et al., 1985; Shimizu and Nakao, 1992). Moreover, although there is autoradiographic evidence for the existence of AVP-binding sites in the neural lobe (Bunn et al., 1986), AVP binding in the neural lobe appears to reflect the presence of neurophysins rather than AVP receptors (Freund-Mercier et al., 1991). Nonetheless, even if AVP receptors are located on AVP or OT terminals in the neural lobe, a direct action of DDAVP at this site would only be likely if those receptors were of the $V_{2}$ type, which has not been reported in neural tissue (Antoni, 1984). Although there is therefore little evidence supporting the likelihood of DDAVP acting directly on AVP and OT neurons at the level of the neural lobe, it is even less 
A
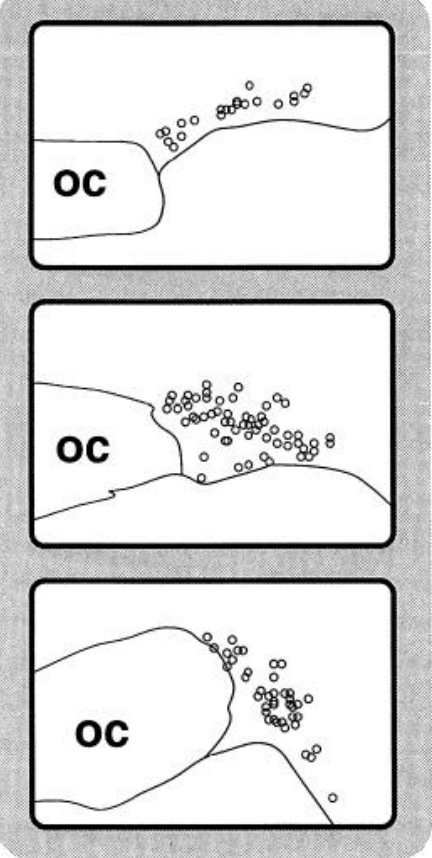

sham
B
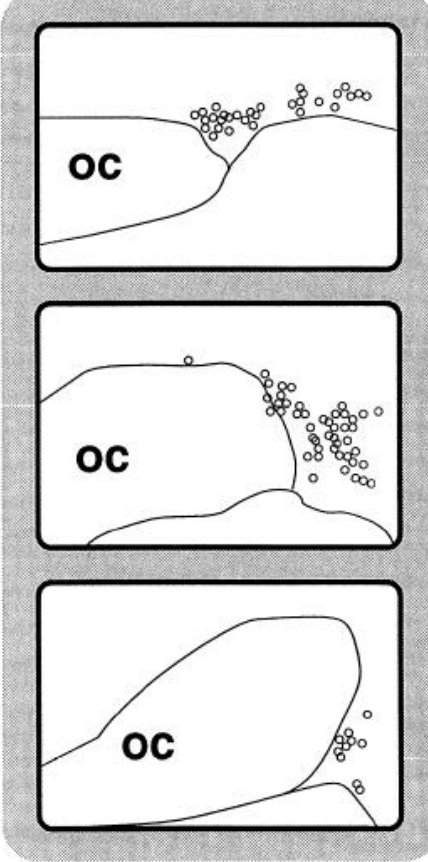

SC/normo
C
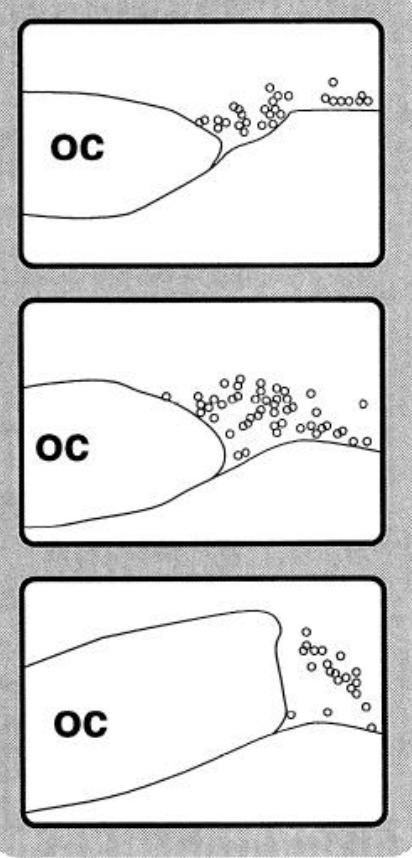

SC/hyper
D
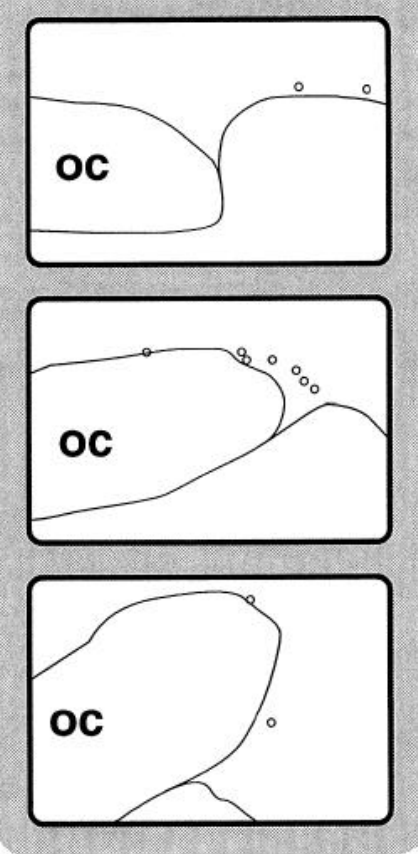

SC/hypo

Figure 7. Topographically correct representation of OT-NP neurons at three different rostrocaudal levels in the SON from a normonatremic sham-operated rat $(A)$, normonatremic SC rat $(B)$, hypernatremic SC rat $(C)$, and hyponatremic SC rat $(D)$. oc, Optic chiasm.

A

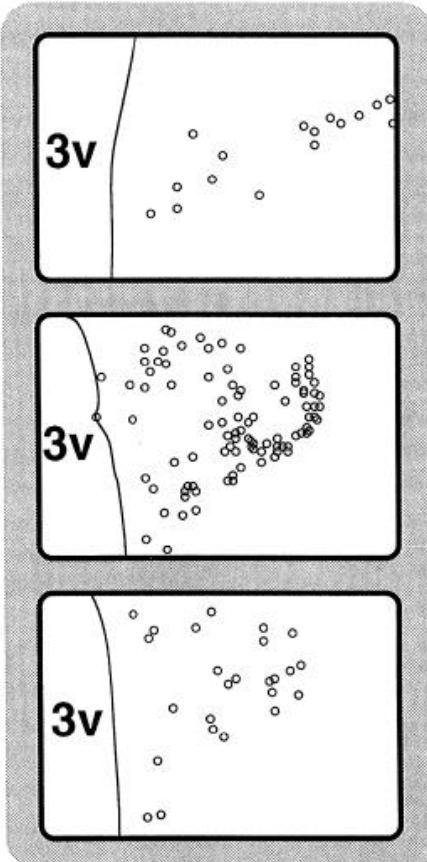

sham
B
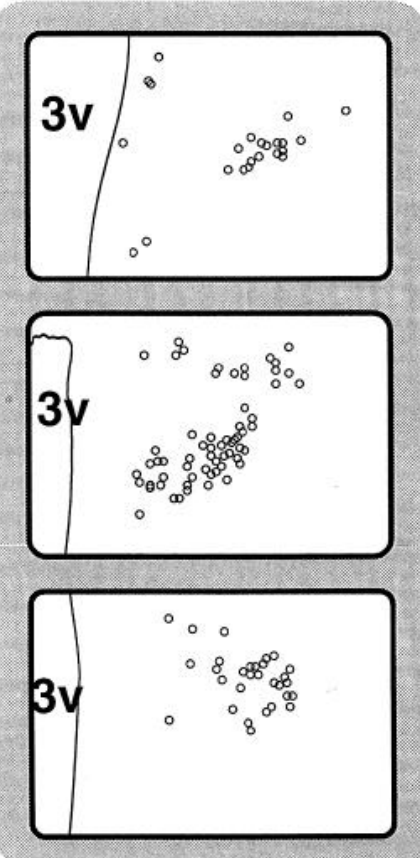

SC/normo
C
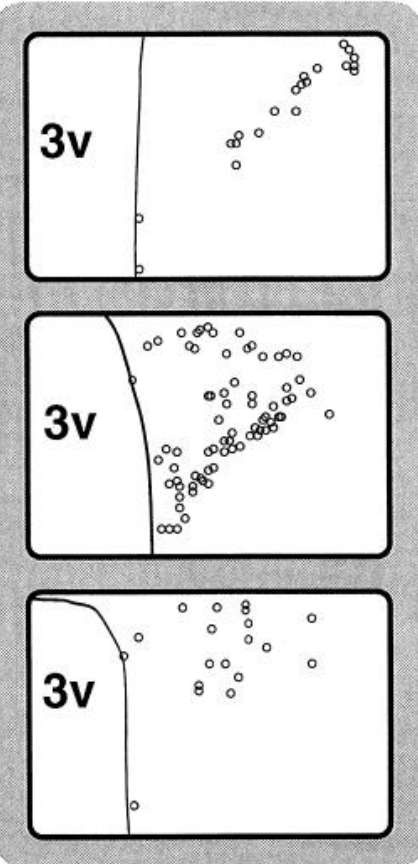

SC/hyper
D
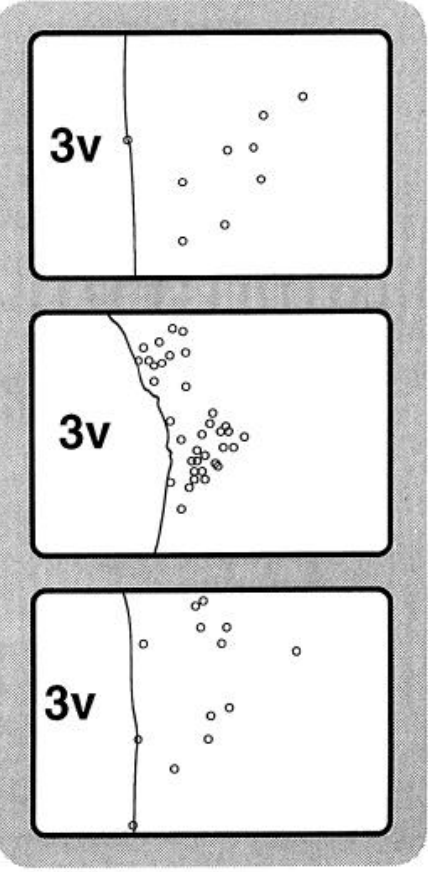

SC/hypo

Figure 8. Topographically correct representation of OT-NP neurons at three different rostrocaudal levels in the SON from a normonatremic sham-operated rat $(A)$, normonatremic SC rat $(B)$, hypernatremic SC rat $(C)$, and hyponatremic SC rat $(D)$. $3 v$, Third ventricle. 
likely that DDAVP could act directly at the level of the perikarya because DDAVP does not cross the blood-brain barrier in appreciable amounts (Stegner et al., 1983); but it is possible that permeability of the blood-brain barrier could have changed in our experiments. It is also theoretically possible that DDAVP could influence magnocellular neurons by acting at circumventricular organs, such as the subfornical organ or the organum vasculosum of the lamina terminalis.

A more likely cause of the excessive loss of magnocellular neurons in the SC hyponatremic rats is the greatly decreased functional activity of magnocellular neurons during induced hyponatremia. During chronic hyponatremia, stimulus-induced AVP and OT secretion is blocked or greatly suppressed (Verbalis et al., 1986; Verbalis and Dohanics, 1991). In addition, in chronically hyponatremic rats, synthesis rates of magnocellular hormones are decreased to $5-25 \%$ of the levels in normonatremic rats (Robinson et al., 1990). Consequently, it seems very likely that the metabolic rates in magnocellular neurons are also much lower during chronic hyponatremia. In support of this, we recently observed that morphological changes in oxytocin axonal structure that normally occur in rats after PVN lesions failed to occur in chronically hyponatremic rats (Dohanics et al., 1993a). A potentially equally important effect of chronic hyponatremia that may be relevant for magnocellular survival rates after SC is the lack of osmotic stimuli that are constantly present under normonatremic conditions. Hyponatremia, therefore, may directly impair the intracellular mechanisms necessary for structural and functional repairs after $\mathrm{SC}$, or these intracellular reparative mechanisms may be compromised by the hyponatremia-induced inactivity of afferent neuronal inputs to magnocellular neurons that contribute to the baseline activity levels of AVP and OT neurons under normonatremic conditions.

Our results clearly showed that hyponatremia significantly decreases the likelihood of magnocellular neuronal survival after axonal injury, but hypernatremia, which is well known to upregulate magnocellular afferent stimulation, synthetic activity, and secretion, did not appear to confer any increased survival advantage. This indicates that the effects of neuronal activity on magnocellular survival are not symmetrical, and suggests that at baseline levels of plasma osmolality optimum conditions for magnocellular regeneration after axonal injury may already exist, in which case hypernatremia would not further enhance the likelihood of cell survival. Perhaps more interesting, however, is the finding that the markedly increased metabolic activity produced by the chronic hyperosmolality did not itself further compromise neuronal survival after SC. However, because hypernatremia lasted only a little over $24 \mathrm{hr}$ after SC, direct comparisons between the effects of hypo- and hyperosmolality on magnocellular survival cannot be extended to the entire postoperative period.

It is also important to note that the results of the cell survival studies are consistent with the functional deficits in neurohypophyseal secretion produced by the SC as reflected by the drinking behavior of the rats. The postoperative triphasic pattern of water intake observed in SC normonatremic rats was essentially identical to that observed in SC hypernatremic rats. In contrast, stalkcompressed hyponatremic rats exhibited a very different pattern of water intake. After surgery, the continued DDAVP infusion prevented any increase in the water intake of these rats despite the loss of their ability to secrete AVP. However, after cessation of DDAVP infusion, the water intakes of these rats increased dramatically and reached levels much higher than those of either $\mathrm{SC}$ normonatremic or SC hypernatremic rats. Furthermore, the peak water intakes observed in SC hyponatremic rats were more than twice as high as the water intakes of Brattleboro rats, which lack AVP immunoreactive neurons (Valtin et al., 1965), and also of neurolobectomized AVP-treated rats in the experiments of Herman et al. $(1986,1987)$. This observation suggests a complete lack of urine-concentrating capability in SC hyponatremic rats. In Brattleboro rats that develop diabetes insipidus early in postnatal life, incrcascd OT sccrctory activity has been suggested to compensate partially for the deficiency of AVP secretion. A similar mechanism could account for the relatively milder polydipsia observed in neurolobectomized AVP-treated rats, which lose most of their AVP magnocellular neurons but appear to retain their OT neuron populations relatively intact (Herman et al., 1987). In contrast, after SC in hyponatremic rats both AVP and OT neuronal populations undergo substantial degeneration, thereby leaving no hormonal secretion capable of exerting antidiuretic effects at the kidneys.

In summary, our results indicate that injury-induced degeneration of magnocellular neurons is amplified by chronic hyponatremia, either by removing osmotic stimuli that would normally impinge on magnocellular neurons or by downregulating the metabolic and synthetic activity of the neurons. These results are potentially relevant for considering treatment strategies for human patients with postoperative or post-traumatic injuries to the hypothalamo-neurohypophyseal system. Such patients are usually treated with DDAVP to provide symptomatic relief of their diabetes insipidus. However, this therapy may in fact be detrimental to long-term survival of the injured AVP and OT neurons by virtue of decreasing the basal activity of these neurons. In this case, prospects for functional recovery of neurohypophyseal secretion might be best enhanced by treatment of ongoing urinary water losses simply by free water replacement, thereby allowing a continual stimulation of the activity of the injured neurons. Further studies will be necessary to understand better the relationship between osmotic status and the regenerative ability of magnocellular neurons, and the cellular mechanisms that are responsible for these effects. Similarly, the degree to which these observations generalize to other neuronal systems is conjectural at this time, but it is interesting to note that a conceptually similar hypothesis regarding the importance of ongoing neuronal activity for neuronal survival has been advanced recently as a possible explanation for the neuronal loss in Alzheimer's disease (Sofroniew, 1991).

\section{REFERENCES}

Abercrombic M (1946) Estimation of nuclear population from microtome sections. Anat Rec 94:239-247.

Antoni FA (1984) Novel ligand specificity of pituitary vasopressin receptors in the rat. Neuroendocrinology 39:186-188.

Antoni FA, Kovács KJ, Dohanics J, Makara GB, Holmes MC, Mazurek MF (1988) Hypophysiotrophic function of vasopressin and oxytocin. Brain Res Bull 20:729-736.

Bodian D, Maren TH (1951) The effect of neuro- and adenohypophysectomy on retrograde degeneration in hypothalamic nuclei of the rat. J Comp Neurol 94:485 514.

Brand S, Mugnaini E (1976) Fulminant Purkinje cell death following axotomy and its use for analysis of the dendritic arborization. Exp Brain Res 26:105-119.

Bunn SJ, Hanley MR, Wilkin GP (1986) Autoradiographic localization of peripheral benzodiazepine, dihydroalprenolol and arginine vasopressin binding sites in the pituitaries of control, stalk transected and Brattleboro rats. Neuroendocrinology 44:76-83. 
Cheng SWT, North WG (1989) Vasopressin reduces release from vasopressin-neurons and oxytocin-neurons by acting on $V_{2}$-like receptors. Brain Res 479:35-39.

Claybauch JR (1976) Effect of dehydration on stimulation of $\triangle D H$ re lease by heterologous renin infusions in conscious dogs. Am J Physiol 231:655-660.

Dohanics J, Hoffman GE, Smith MS, Verbalis JG (1992) Functional neurolobectomy induced by controlled compression of the pituitary stalk. Brain Res 575:215-222.

Dohanics J, Hoftman GE, Verbalis JG (1993a) Hyponatremia inhibits sprouting of oxytocinergic axons following bilateral lesions of the paraventricular nucleus in rats. Neurosci Lett 165:51-54.

Dohanics J, Hoffman GE, Verbalis JG (1993b) C-Fos and C-Jun expression in the hypothalamic magnocellular neurons after pituitary stalk injury. Soc Neurosci Abstr 19:530.

Freund-Mercier MJ, Stoeckel ME, Waeber C, Kremarik P, Palacios JM, Richard PH (1991) Neurophysins, rather than receptors, are involved in $\left[{ }^{3} \mathrm{H}\right]$ oxytocin and $\left[{ }^{3} \mathrm{H}\right]$ vasopressin binding detected by autoradiography in the hypothalamo-neurohypophyseal system. J Neuroendocrinol 3:285-295.

Fry FJ, Cowan WM (1972) A study of retrograde cell degeneration in the lateral mamillary nucleus of the cat, with special reference to the role of axonal branching in the preservation of the cell. J Comp Neurol 144:1-24.

Grothe C, Otto D, Unsicker K (1989) Basic tibroblast factor promotes in vitro survival and cholinergic development of rat scptal neurons: comparison with the effects of nerve growth factor. Neuroscience $31: 649-661$

Hare K (1937) Degeneration of the supra-optic nuclcus following hypophysectomy in the dog. Am .I Physiol 119:326.

Hefti F (1986) Nerve growth factor promotes survival of septal cholinergic neurons after fimbrial transections. J Neurosci 6:2155-2162.

Herman JP, Marciano FF, Gash DM (1986) Vasopressin administration prevents functional recovery of the vasopressinergic ncurosecretory system following neurohypophysectomy. Neurosci Lett 72:239-246.

Herman JP, Marciano FF, Wiegand SJ, Gash DM (1987) Selective cell death of magnocellular vasopressin neurons in ncurohypophysectomized rats following chronic administration of vasopressin. J Neurosci $7: 2564-2575$.

Kawaguchi S, Miyata H, Kawamura M, Harada Y (1981) Morphological and electrophysiological evidence for axonal regeneration of axotomized cerebellothalamic neurons in kittens. Neurosci Lett 25:13-18.

I undin S, Melin P, Vilhardt H (1985) Use of three specific radioimmunoassays in measuring neurohypophysial hormone content and plasma concentrations of vasopressin, oxytocin and DDAVP in rats after prolonged infusion of DDAVP. Experientia 41:933-935.

Marciano FF, Wiegand SJ, Sladek Jr JR, Gash DM (1989) Fetal hypothalamic transplants promote survival and functional regeneration of axotomized adult supraoptic magnocellular neurons. Brain Res $135-142$.

Moll J, De Wied D (1962) Observations on the hypothalamoposthypophyseal system of the posterior lobectomized rat. Gen Comp Endocrinol 2:215-228.
O'Connor WI (1947) Atrophy of the supraoptic and paraventricular nuclei after interruption of the pituitary stalk in dogs. Q J Exp Physiol $34: 29-42$.

O'Connor WJ (1952) The normal interphase in the polyuria which follows section of the supraoptico-hypophysial tracts in the dog. Q J Exp Physiol 37:1-10.

Raisman G (1973) An ultrastructural study of the effects of hypophysectomy on the supraoptic nucleus of the rat. J Comp Neurol 147:181-208.

Robinson AG, Roberts MM, Evron WA, Verbalis JG, Sherman TG (1990) Hyponatremia in rats induces downregulation of vasopressin synthesis. J Clin Invest 86:1023-1029.

Sherman TG, McKelvy JF, Watson SJ (1986) Vasopressin mRNA regulation in individual hypothalamic nuclei: a Northern and in situ hybridization analysis. J Neurosci 6:1685-1694.

Shimizu K, Nakao A (1992) Effect of 1-desamino-8-D-arginine vasopres$\sin$ (DDAVP) on vasopressin release and blood pressure during hemorrhage. Endocrinol Jpn 39:517-523.

Sievers J, Hausmann B, Berry M (1989) Fetal brain grafts rescue adult retinal ganglion cells from axotomy-induced cell death. J Comp Neurol 281:467-478

Sofroniew MV (1991) Can activity modulate the susceptibility of neurons to degeneration? Neurobiol Aging 12:351-352.

Sofroniew MV, Isacson O (1988) Distribution of degeneration of cholinergic neurons in the septum following axotomy in different portions of the fimbria-fornix: a correlation between degree of cell loss and proximity of neural somata to the lesion. J Chem Neuroanat 1:327-337.

Stegner H, Artman HG, Leake RD, Fisher DA (1983) Does DDAVP (1-desamino-8-D-arginine-vasopressin) cross the blood-CSF barrier? Neuroendocrinology 37:262-265.

Tuszynski MH, U HS, Amaral DG, Gage FH (1990) Nerve growth factor infusion in the primate brain reduces lesion-induced cholinergic neuronal degeneration. J Neurosci 10:3604-3614.

Valtin H, Sawyer WH, Sokol HW (1965) Neurophysiological principles in rats homozygous and heterozygous for hypothalamic diabetes insipidus (Brattleboro strain). Endocrinology 77:701-706.

Verbalis JG, Dohanics J (1991) Vasopressin and oxylucin secretion in chronically hypoosmolar rats. Am J Physiol 261:R1028-R1038.

Verbalis JG, Drutarosky MD (1988) Adaptation to chronic hypoosmolality in rats. Kidncy Int 34:351-360.

Verbalis JG, Baldwin EF, Robinson AG (1986) Osmotic regulation of plasma vasopressin and oxytocin after sustained hyponatremia. Am J Physiol 250:R444-R451.

Verbalis JG, Martinez AJ, Drutarosky MD (1991a) Neurological and neuropathological sequeale of correction of chronic hyponatremia. Kidney Int 39:1274-1282.

Verbalis JG, Stricker EM, Robinson AG, Hoffman GE (1991b) Cholecystokinin aclivales C-Fos expression in hypothalamic oxytocin and corticotropin-releasing hormone neurons. J Neuroendocrinol 3:205-213.

Yu WA (1989) Administration of testosterone attenuates neuronal loss following axotomy in the brain-stem motor nuclei in female rats. J Neurosci 9:3908-3914. 